\title{
Preparation of Fluoroalkyl-containing Silica Nanoparticles and Its Waterproofing Application on Concrete
}

\author{
Zhigang Zhu ${ }^{1,}$, Lisong $\mathrm{Hu}^{1}$, Qingzhong Guo ${ }^{2}$ \\ ${ }^{1}$ School of Resource and Civil Engineering, Wuhan Institute of Technology, Wuhan 430073, China \\ ${ }^{2}$ School of Materials Science and Engineering, Wuhan Institute of Technology, Wuhan 430073, \\ China \\ aemail: Ishu123a@sina.cn
}

Keywords: Fluorine-containing organosilane; Hydrophobicity; Water-repellent Agent; Nanoparticles

\begin{abstract}
Fluoroalkyl-containing silica nanoparticles were prepared and characterized by a combination of IR and TEM analysis. The obtained silica nanoparticles with an average diameter 40 $\mathrm{nm}$ were coated onto concrete. Surface properties of the coated concrete were characterized by water contact angle (WCA) and X-ray photoelectron spectroscopy (XPS). The fluoroalkyl groups migrated preferentially towards air-film interface to generate a hydrophobic surface with a WCA of $140.3^{\circ}$ and the ratio of absorbing water was $5.6 \%$. These results demonstrated that the fluoroalkyl-containing nanomaterials could be used as water-repellent surface-treating agent for concrete and had excellent waterproofing performance.
\end{abstract}

\section{Introduction}

Surface-treatment of cement and concrete has been regarded as an effective way of pursuing a longer service life of construction structure [1]. In the past several decades, organosilicon materials have been widely used as surface-treatment water-repellent agents in the field of construction due to their attractive characteristics, such as low-surface-energy, hydrophobicity, weatherability, and widespread applicability. They are regarded as effective waterproof agents because they can make the surface of construction hydrophobic, thus reduces the intake of aggressive agents. However, most of organosilicon water-repellent agents are containing methyl or alkyl group which are lipophilic and lead to poor anti fouling performance of surface. In order to improve the anti fouling performance, fluorine-containing organosilicon materials have been used instead of methyl or alkyl group silicon water-repellent agent [2, 3]. The fluoroalkyl groups could migrate preferentially towards air-film interface and make the surface with predominant hydrophobicity and oleophobicity [4]. In this article, fluoroalkyl-containing silica nanoparticles were synthesized and characterized and then coated onto concrete. The waterproofing performance of the resultant fluoroalkyl-containing nanomaterials was also evaluated.

\section{Experimental}

Triethoxy-1H,1H,2H,2H-tridecafluoro-n-octylsilane (F-8261) was purchased from Degussa Co. (Germany). Other reagent-grade solvents and chemicals were used as received.

Infrared (IR) spectra were obtained in the 4000-400 $\mathrm{cm}^{-1}$ region using a Bio-Rad FTS infrared spectrophotometer with $\mathrm{KBr}$ pellet technique. Transmission electron microscopy (TEM) observations were performed with an H7650B system (Hitachi, Japan) under an acceleration voltage of $20 \mathrm{kV}$. Contact angles (CAs) were evaluated at room temperature on the Krüss DSA100 instrument, equipped with a video camera. Water was adopted for the analysis and the volume of water droplet was $2 \mu \mathrm{L}$. X-ray photoelectron spectroscopy (XPS) measurement was carried out using a Kratos Axi Ultra HAS photoelectron spectrometer. The bare pressure in the chamber was maintained at $4.0 \times 10^{-9} \mathrm{~Pa}$ during the measurement.

Preparation of fluorine-containing silica nanoparticles $\left(\mathrm{F}-\mathrm{SiO}_{2}\right)$ :

Silica nanoparticles of mean diameter $(40 \mathrm{~nm})$ were prepared by hydrolysis of tetraethyl 
orthosilicate (TEOS) in an alcohol medium in the presence of water, sodium hydrate and tetramethylammonium hydroxide by a modified procedure, originally described by stöber et al [5]. The reaction was ended by adding acid to neutralize the catalyst. Then, F-8261 was added to a 50 $\mathrm{mL}$ portion of the silica nanoparticles colloid (the volume ratio of F-8261 and TEOS was 1:15) while vigorously stirring and allowed to react for $6 \mathrm{~h}$. The F-8261-functionalized silica nanoparticles were purified by centrifuging and redispersing in ethanol.

Waterproofing Application of F-SiO ${ }_{2}$ on Concrete:

The preparation of concrete sample and the test procedure for the ratio of absorbing water were under the condition of JC/T 902-2002 (China). Before surface treatment with $\mathrm{F}^{-\mathrm{SiO}_{2}}$ by brushing technique, the concrete samples were dried at $60{ }^{\circ} \mathrm{C}$ for 1 day. The surface was brushed for three times at a time-interval of $10 \mathrm{~min}$.

\section{Results and Discussion}

The silica nanoparticles with an average diameter $(40 \mathrm{~nm})$ were prepared using the sol-gel process. Fluorine-containing silica nanoparticles were prepared by reaction of the obtained nano-silica and F-8261. Figure 1 shows representative TEM images of nano- $\mathrm{SiO}_{2}$ and $\mathrm{F}-\mathrm{SiO}_{2}$. The particle sizes were relatively uniform and it is evident that the modification of nano-silica with F-8261 molecules has little effect on the size and morphology of silica nanoparticles as determined by TEM images.
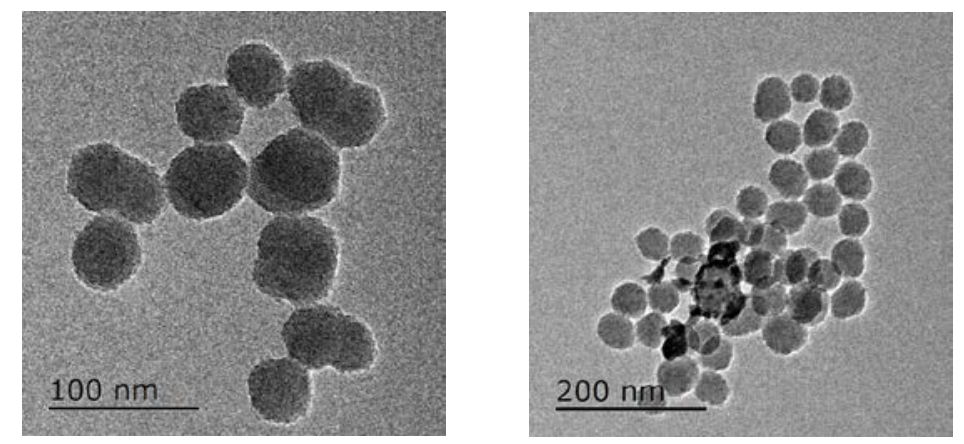

Fig. 1. TEM images of nano-silica (left) and fluorine-containing silica nanoparticles (right)

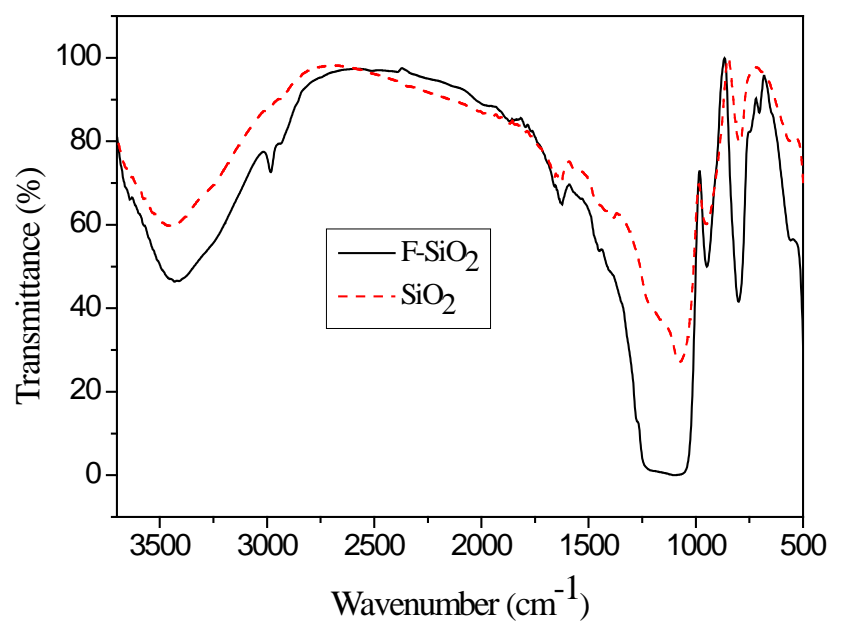

Fig. 2. FT-IR spectra of nano-silica $\left(\mathrm{SiO}_{2}\right)$ and fluorine-containing silica nanoparticles

IR spectroscopy was used to confirm the structure of nano-silica and $\mathrm{F}_{-} \mathrm{SiO}_{2}$ and the results were shown in Fig. 2. Nano-silica exhibited the characteristic absorption peak of $\mathrm{Si}-\mathrm{O}$ stretching vibration at about $1050 \mathrm{~cm}^{-1}$, and the broad band which appeared around $3500-3000 \mathrm{~cm}^{-1}$ corresponded to the $\mathrm{O}-\mathrm{H}$ stretching vibration of silica. IR spectrum of $\mathrm{F}-\mathrm{SiO}_{2}$ confirmed that $1 \mathrm{H}, 1 \mathrm{H}, 2 \mathrm{H}, 2 \mathrm{H}$-decafluoro-n-octyl groups had been attached onto the surface of nano-silica. The peaks at 2950 and $2860 \mathrm{~cm}^{-1}$ could be assigned to the antisymmetrical and symmetrical stretching 
vibrations of $\mathrm{C}-\mathrm{H}$, which agreed well with the reported literature values [6]. The $\mathrm{C}-\mathrm{F}$ stretching vibration is normally observed in the wavenumber range $1020-1250 \mathrm{~cm}^{-1}$ [7]. However, this peak was not resolved due to the overlay with the IR absorptions of $\mathrm{Si}-\mathrm{O}-\mathrm{Si}$ in the range 1130-1000 $\mathrm{cm}^{-1}$ and of $\mathrm{Si}-\mathrm{CH}_{2}-\mathrm{R}$ in the range $1250-1200 \mathrm{~cm}^{-1}$. Nevertheless, the peak in this region was broader, indicating possible overlap of peaks.
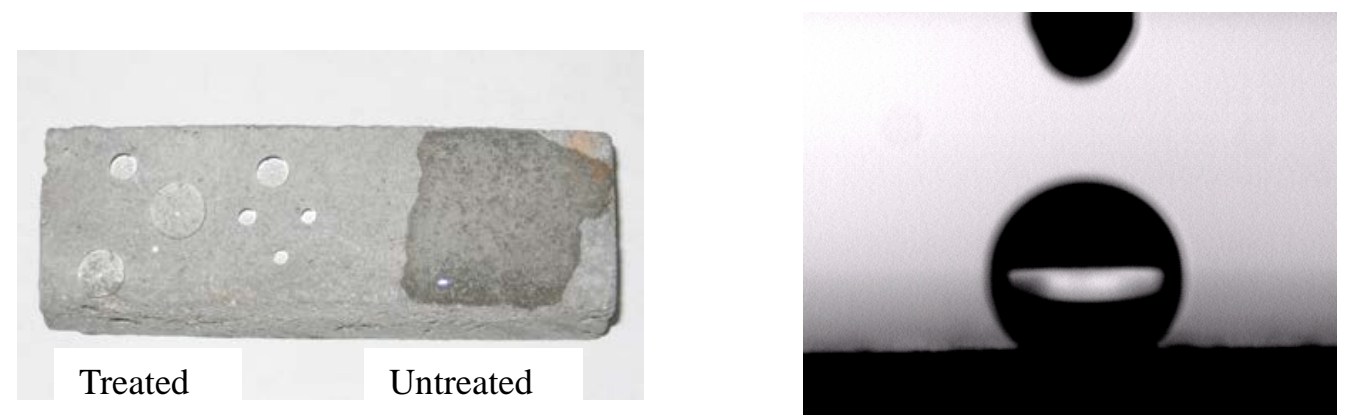

Fig. 3. Photos of water on the surface of concrete treated by F-SiO 2 (left) and water contact angle analysis (right)

The surface chemical composition played an important role on the surface properties of substrate materials. When the concrete sample was treated by $\mathrm{F}_{-} \mathrm{SiO}_{2}$, the fluoroalkyl groups migrate preferentially towards air-film interface to minimize the interfacial energy and generate a superhydrophobic surface [4]. Seen from Fig. 3 (left), the treated surface of concrete was hydrophobic, different from the untreated surface. The non-wettable nature of the surface was evaluated through water contact angle (WCA) measurement. Fig. 3 shows that the surface of concrete sample treated by $\mathrm{F}-\mathrm{SiO}_{2}$ exhibited superhydrophobic nature with a WCA of $140.3^{\circ}$. The ratio of water absorption rate has been widely used to reflect the performance of water-repellent materials. The ratio of absorbing water is $5.6 \%$, which suggested that $\mathrm{F}_{-} \mathrm{SiO}_{2}$ had excellent waterproofing performance. Furthermore, the surface chemical compositions of concrete sample coated with $\mathrm{F}_{-} \mathrm{SiO}_{2}$ were analyzed by XPS. The F1s signal was centered at $688.3 \mathrm{eV}$ (Fig. 4). The tested mass concentration of $\mathrm{F}$ was higher than the theoretical value, which was attributed to the already proved migration property of fluoroalkyl segments.

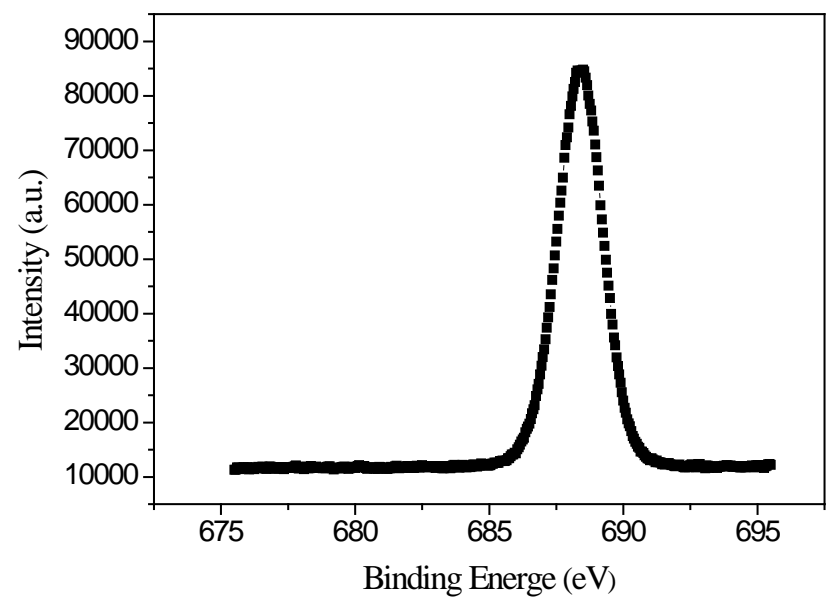

Fig. 4. XPS spectrum of F-SiO 2 coated concrete sample.

\section{Conclusion}

In the present study, a simple method was proposed for the synthesis of fluorine-containing silica nanoparticles. The as-obtained material demonstrated to be used as water-repellent surface-treating agent for concrete and had excellent waterproofing performance. The surface of concrete treated by fluorine-containing silica nanoparticles exhibited hydrophobic nature. 


\section{Acknowledgement}

In this paper, the research was sponsored by Doctoral Program Foundation of Wuhan Institute of Technology (No. 11105032).

\section{References}

[1] Cai Yamei, Hou Pengkun, Duan Ce. The use of tetraethyl orthosilicate silane (TEOS) for surface-treatment of hardened cement-based materials: a comparison study with normal treatment agents, Construction and Building Materials, 2016, 117, 144-151.

[2] Yoneda Takashige, Ohharu Kazuya. Water-repellent surface-treating agents of fluorine-containing silicon compounds, JP, 11116943. 1999.

[3] Campostrini R, Ischia M, Armelao L. Pyrolysis study of fluorinated sol-gel silica. Journal of Thermal Analysis and Calorimetry, 2004, 78(2), 657-677.

[4] Xu Wei, An Qiufeng, Hao Lifen. Synthesis of self-crosslinking fluorinated polyacrylate soap-free latex and its waterproofing application on cotton fabrics, Fibers and Polymers, 2014, 15(3), 457-464.

[5] Wan Peter Hus, Yu Rongchi. Paper whiteners: I, titania coated silics. Journal of Colloid and Interface Science, 1993, 156, 56-65.

[6] Jradi K, Laour D, Daneault C, Chabot B. Control of the chemical and physical behavior of silicon surfaces for enhancing the transition from hydrophilic to superhydrophobic surfaces, Colloid Surface A, 2011, 374, 33-41.

[7] Cai Lu, Dai Li, Yuan Yanhua, Liu Anqi, Li Zhanxiong. Synthesis of novel polymethacrylates with siloxyl bridging perfluoroalkyl side-chains for hydrophobic application on cotton fabrics, Applied Surface Science, 2016, 371, 453-467. 